\title{
A New Robust Pre-Processing Technology of Image Compression based on Wavelets Decomposition
}

\author{
Garima \\ Research Scholar, \\ Department of Computer Science \& Engineering \\ IET, Alwar, India
}

\author{
Deepak Chaudhary \\ Assistant Professor \\ Department of Computer Science \& Engineering \\ IET, Alwar, India
}

\begin{abstract}
Wavelet transform is becoming more and more important in image compression applications because of its suppleness in on behalf of non-stationary signals. Current techniques use comprehensive search trial that are computationally rigorous to find the "optimal" basis. In this paper, we offer some guiding principle based on the statistics of the image to be coded, for formative the need to search for the "optimal" wavelets as well as the development that can be achieved by using the "optimal" over "standard" wavelets. The proposed guidelines can be used to find a good initial guess for the exhaustive search if the optimal basis is needed.
\end{abstract}

\section{Keywords}

Image, wavelets, transform, compression

\section{INTRODUCTION}

Digital image data are capacious and hence dictate the use of compression techniques for storage and transmission. The International Standard Organisation (ISO) has freshly proposed the JPEG and MPEG standards for image and video compression, respectively. These principles utilize the discrete cosine transform (DCT) for intra-image compression. However, DCT has the drawbacks of blackness and aliasing distortions in the reconstructed images at low bit rates. Recently, wavelet transform has become accepted in image and video applications since the basic functions match the human visual characteristics. Wavelet coding techniques result in individually agreeable images due to the deficiency of overcrowding effects and aliasing distortion. In addition, features like scalability can be easily implemented in the wavelet domain. Significant efforts have been directed towards selecting the most favourable filter bank in an image coder $[1,2,3]$. In most cases, the optimal wavelet is found over the whole image. Few cases have been reported for selecting spatially adapted filter banks. Some authors have also reported using different wavelets in different decomposition stages. Normally, finding an optimal wavelet for a particular image is a compute concentrated task. Tewfik et aZ.[2] have proposed a technique to find the best wavelet basis by maximizing the L2-norm of the wavelet approximated signal (up to a given scale). Cagler er d[3] have planned a technique based on optimizing various filter characteristics which are known to affect the coding performance. The difficulty of the algorithm increases exponentially with the order of the filter.

The presentation of wavelet-based coding also depends on the decomposition (tree) structure. Ramachandran et aZ.[4] have projected a technique, based on Lagrangian optimization, to find the best basis subtree, which minimizes the global distortion for a given bit budget. However, this algorithm is also computationally expensive.

\section{IMAGE PRE-TREATMENT WITH DWT TRANSFORM \\ 2.1 The Discrete Wavelet Transform}

The DWT provides adequate in sequence for the analysis and synthesis of a signal, but is favourably, much more efficient. Discrete Wavelet analysis is computed using the concept of filter banks. B. Compression techniques there are many different forms of data compression [9]. This examination will deliberate on change coding and then more exclusively on Wavelet Transforms. Image data can be represented by coefficients of discrete image transforms. Coefficients that make only small assistance to the information contents can be omitted. However wavelets transform is applied to entire images, rather than sub images, so it produces no blocking artefacts. This is a major advantage of wavelet compression over other transform compression methods. An important point to note about Wavelet compression is explained by Aboufadel[3]: "The use of wavelets and thresholding serves to process the original signal, but, to this point, no actual compression of data has occurred". This explains that the wavelet analysis does not actually compress a signal, it simply provides information about the signal which allows the data to be compressed by ISOM which use standard entropy coding techniques, such as Huffman coding. Huffman coding is good to use with a signal processed by wavelet analysis, because it relies on the fact that the data values are small and in particular zero, to compress data. It works by giving large numbers more bits and small numbers fewer bits. Long strings of zeros can be encoded very efficiently using this scheme. Therefore an actual percentage compression value can only be stated in conjunction with an entropy coding technique.

\subsection{Wavelet and Compression}

Wavelets are helpful for compressing signals but they also have far more widespread uses. They can be used to process and pick up signals, in fields such as gray scale image. They can be used to remove noise in an image, for example if it is of very fine scales, wavelets can be used to cut out this fine scale, effectively removing the noise.

A significant illustration of visual data is the main focus of several image processing techniques such as compression, denoising, feature extraction, etc. competence of a representation is the potential to capture substantial information about an object of interest through a small description. For image compression, the application of a competent representation shows the solidity of the compressed file or the index entry for each image in the database. This efficient symbol can be attained through competent transforms and fast algorithms [4]. There are five very necessary properties of image depiction which are to be taken into consideration [5]. 
- Multiresolution: The illustration is very closely related to the image when multi-resolution starting from a low resolution to a higher resolution

- Localization: Base mechanism should be in both spatial and frequency domain.

- Critical Sampling: the frame or redundancies for the base must be small which is created by symbol.

- Directional: different regions in good representation must have base elements.

- Anisotropy: To obtain the smooth contours in an image, base elements in depiction can have long shapes with dissimilar wide-length ratios.

The first three properties are obsessed by wavelet, while first four properties are obsessed by edge lets but contour let possess all properties. The Wavelet-Based Contourlet Transform (WBCT) is used to improve the overall presentation of contour let transform. It is mostly used for change of image coding. The method of assemblage an incessantly valued input data, to collected works of discrete valued output data is called as quantization [6,7]. The quality and varying levels of image compression are achieved through by selecting different quantization levels. The individual coefficients in scalar quantization are assisted to be transformed to a quantized with the alteration changes from coefficient to the coefficient. In this paper scalar quantization is used in its place of scalar quantization along with waveletbased contour let transformation for better results.

\section{METHODOLOGY}

The two coding techniques that are being compared are the Discrete Cosine Transform (DCT) and Discrete Wavelet Transform (DWT). The DCT-based encoder worked by segmentation of the image into $8 * 8$ blocks.

\subsection{The Discrete Cosine Transform (DCT)}

DCT has been successfully employed as the first step in several coding system, because it exhibits favourable energy distribution in the frequency domain. DCT attempts to decorrelate the image data and after, each transform coefficient can be encoded without dropping off compression efficiency.

\subsubsection{Algorithm Al (DCT Encoding System)}

Step1. Load image

Step2. The image is broken into $\mathrm{N}^{*} \mathrm{~N}$ blocks of pixels. Here $\mathrm{N}$ may be $4,8,16$, e t $\mathrm{c}$.

Step3. Working from left to right, top to bottom, the DCT is applied to each block.

Step4. Each block's elements are compressed through quantization means dividing by some specific value.

Step5. The array of compressed blocks that constitute the image is stored in a drastically reduced amount of space.

The whole image is divided into small $\mathrm{N}^{*} \mathrm{~N}$ blocks then DCT is applied on these blocks. For reducing the storage space, DCT coefficients are quantized through dividing by some value or by quantization matrix; so that large value become small and it needs small size of space.

\subsubsection{Algorithm A2 (DCT Decoding System)}

Step1. Load compressed image from disk

Step2. Image is broken into $\mathrm{N} * \mathrm{~N}$ blocks of pixels.
Step3. Each block is de-quantized by applying reverse process of quantization.

Step4. Now apply inverse DCT on each block. And combine these blocks into an image which is identical to the original image.

In the decoding process, N's value was kept the same as it used in encoding process. Then dequantization process was performed by multiplying with quantization value or quantization matrix. As earlier said that this is lossy technique so output image is not exact copy of original image but it resembled the original image.

\subsection{Discrete Wavelet Transform (DWT)}

There are two types of wavelet in used. First one is wavelet transform and second one is discrete wavelet transform. Wavelet analysis is computed by filter bank.

\subsubsection{Algorithm B1 (DWT Encoding System)}

Step1. First original image have to been passed through high pass filter and low pass filter by applying filter on each row.

Step2. Output of the both filtered images are combined into T1

Step3. T1 is down sampled by 2 .

Step4. Again, T1 has been passed through high pass filter and low filter by applying on each column.

Step5. Output of the step4 is passed through high pass filter and low pass filter again and then combined into T3

Step6. Lastly, down sample T3 by 2 .This is our compressed image.

\subsubsection{Algorithm B2 (DWT Decoding System)}

Step1. Extract low pass filter image and high pass filter image from compressed image.

Step2. Both images are up sampled by 2 .

Step3. Now we take the summation of both images into one image called R1.

Step4. Again extract low pass filter image and high pass filter image by simply dividing vertically. First half is low pass filtered image. And second half is high pass filter image.

Step5. Take summation of both images. That is our reconstructed image.

Though in DWT, very high compression ratio was gotten, and minimum amount of information was lost, but, more than one level was done, then more compression ratio was gotten and the reconstructed image is not identical to original image.

\subsection{Haar Transform}

Haar functions have been used from 1910 when they were introduced by the Hungarian mathematician Alfred Haar [10]. Haar wavelet is discontinuous, and resembles a step function. It represents the same wavelet as Daubechies db1. Haar used these functions to give an example of an orthonormal system for the space of square-integrable function on the unit interval $[0,1]$. 


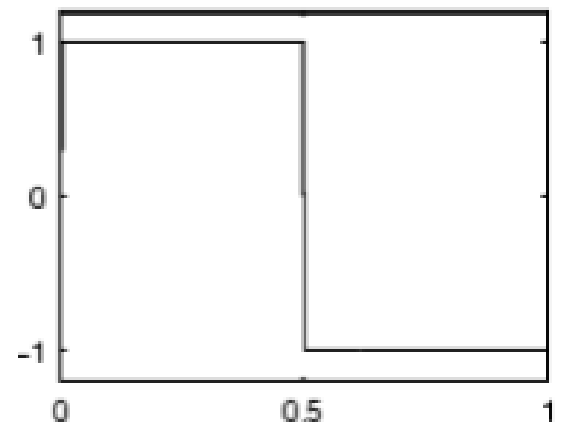

Fig 1: Haar wavelets

For an input represented by a list of numbers, the Haar wavelet transform may be measured to simply pair up input values, storing the difference and passing the sum. This process is frequent recursively, combination up the sums to offer the next scale, finally resultant in differences and one final sum. The Haar Wavelet Transformation is a simple form of compression which involves averaging and differencing terms, storing detail coefficients, eliminating data, and reconstructing the matrix such that the resulting matrix is similar to the initial matrix.[11-12]. A Haar wavelet is the simplest type of wavelet. In discrete form, Haar wavelets are related to a mathematical operation called the Haar transform. The Haar transform serves as a prototype for all other wavelet transforms. Like all wavelet transforms, the Haar transform decomposes a discrete signal into two sub-signals of half its length. One sub-signal is a running average or trend; the other subsignal is a running difference or fluctuation.

\subsection{Daubechies Transform}

Ingrid Daubechies, one of the brightest stars in the world of wavelet research, invented what are called compactly supported orthonormal wavelets - thus making discrete wavelet analysis practicable. The names of the Daubechies family wavelets are written $\mathrm{dbN}$, where $\mathrm{N}$ is the order, and $\mathrm{db}$ the - surnamell of the wavelet. The db1 wavelet, as mentioned above, is the same as Haar wavelet. Here is the wavelet functions psi of the next nine members of the family.

The Daubechies wavelet transforms are defined in the same way as Haar wavelet transform by computing the running averages and differences via scalar products with scaling signals and wavelets the only difference between them consists in how these scaling signals and wavelets are defined[13]. This wavelet type has balanced frequency responses but non-linear phase responses. Daubechies wavelets use overlapping windows, so the high frequency coefficient spectrum reflects all high frequency changes. Therefore Daubechies wavelets are useful in compression and noise removal of audio signal processing.

\section{CONCLUSIONS}

Image is an important medium for people to transmit information, and the large amount of data is a distinctive feature of a digital image, so the image compression is very important for the rapid growth of information today. This paper uses the wavelet transform for image compression, introduces the basic theory of wavelet transform and thoughts of wavelet transform in image compression. At the same time, it details the two different methods of image compression: rounding high frequency, taking low frequency and the threshold method. For threshold method, this paper uses global threshold compression and hierarchical threshold compression, and according to the experimental results, it can get the conclusion which under the condition that the energy loss is not large, threshold compression can obtain higher compression In this paper, the results of different waveletbased image compression techniques are compared. The effects of different wavelet functions, filter orders, number of decompositions, image contents and compression ratios are examined.

\section{REFERENCES}

[1] Digital Image Processing (Pearson Education, Second Edition) By Rafael C. Gonzalez and Richard E.Woods.

[2] Digital Image Processing by Athur R.Weak

[3] Digital Image Processing using MATLAB (Pearson Education) By Rafael C. Gonzalez, Richard E. Woods and Steven L.Eddins,

[4] T Hong LIU , Lin-pei ZHAIV , Ying GAO, Wen-ming

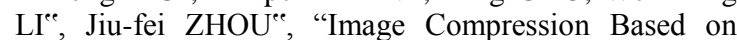
BiorthogonalnWavelet Transform", IEEE Proceedings of ISCIT2005

[5] De Vore , et al.,n"Image Compression through Wavelet Transform Coding", IEEE Transaction on Information Theory.

[6] "A Comparative Study of Image Compression Techniques Based on Svd, Dwt-Svt , Dwt-Dct" ICSCI2008 proceedings pg 494-496

[7] R. Edmund, -Wavelet Compression of Medical Imageryll. Tele-medicine Journal, Vol. 2, 1996, pp. 131137.

[8] Mamta Sharma, compression Using Huffman Coding, IJCSNS International Journal of Computer Science and Network Security, VOL.10 NO.5, May 2010,pp 133-141

[9] Mahendra V.P., Arjun M.R., Yadav H.S., Manoj M.S. "Implementation of advance Image Compression Algo. using WT" (http://sidharth0384.googlepages.com/ Report.pdf)

[10] Nanavati S. P. and Panigrahi P.K., 2005. "Wavelet: Applications to Image Compression-II" (http://www.ias.ac.in/resonance/Mar2005/pdf/Mar2005p 19-27.pdf)

[11] Sindhu M., Rajkamal R., 2009. "Image and its compression techniques" International Journal of Recent Trends in Engineering, Vol 2, No. 4, pp. 71-75.

[12] Kumar, T. and K. Verma, 2010a. A theory based on conversion of RGB image to gray image. Int. J. Computer. Appli., 7: 5-12. DOI: 10.5120/1140-1493 